\title{
Quantitative imaging values of CT, $M R$, and FDG-PET to differentiate pineal parenchymal tumors and germinomas: are they useful?
}

\section{$\operatorname{AUTHOR}(S)$ :}

Kakigi, Takahide; Okada, Tomohisa; Kanagaki, Mitsunori; Yamamoto, Akira; Fushimi, Yasutaka; Sakamoto, Ryo; Arakawa, Yoshiki; ... Shimono, Taro; Takahashi, Jun C; Togashi, Kaori

\section{CITATION:}

Kakigi, Takahide ... [et al]. Quantitative imaging values of CT, MR, and FDG-PET to differentiate pineal parenchymal tumors and germinomas: are they useful?. Neuroradiology 2014, 56(4): 297-303

\section{ISSUE DATE:}

2014-02-09

\section{URL:}

http://hdl.handle.net/2433/201495

\section{RIGHT:}

The final publication is available at Springer via http://dx.doi.org/10.1007/s00234-0141334-2.; This is not the published version. Please cite only the published version.; この論 文は出版社版でありません。引用の際には出版社版をご確認ご利用ください。 


\section{Quantitative Imaging Values of CT, MR and FDG-PET to Differentiate Pineal Parenchymal Tumors and Germinomas: Are They Useful?}

Takahide Kakigi, $\mathrm{MD}^{1)}$, Tomohisa Okada, $\mathrm{MD}, \mathrm{PhD}^{1)^{*}}$, Mitsunori Kanagaki, $\mathrm{MD}$, $\mathrm{PhD}^{1)}$, Akira Yamamoto, $\mathrm{MD}, \mathrm{PhD}^{1)}$, Yasutaka Fushimi, $\mathrm{MD}, \mathrm{PhD}^{1)}$, Ryo Sakamoto, $\mathrm{MD}^{1)}$, Yoshiki Arakawa, $\mathrm{MD}, \mathrm{PhD}^{2)}$, Yoshiki Mikami, $\mathrm{MD}, \mathrm{PhD}^{3)}$, Taro Shimono, $\mathrm{MD}$, $\mathrm{PhD}^{4)}$, Jun C. Takahashi, $\mathrm{MD}, \mathrm{PhD}^{2)}$, and Kaori Togashi, $\mathrm{MD}, \mathrm{PhD}^{1)}$

1) Department of Diagnostic Imaging and Nuclear Medicine, ${ }^{2)}$ Department of Neurosurgery, and ${ }^{3)}$ Department of Pathology, Kyoto University Graduate School of Medicine, Kyoto, Japan. ${ }^{4)}$ Department of Radiology, Osaka City University Graduate School of Medicine, Osaka, Japan.

Corresponding Author: Tomohisa Okada, MD, PhD

Department of Diagnostic Imaging and Nuclear Medicine,

Kyoto University Graduate School of Medicine,

54 Shogoin Kawahara-cho, Sakyo-ku, Kyoto, 606-8507, Japan

Phone: +81-75-751-3760, FAX: +81-75-771-9709 
E-mail: tomokada@kuhp.kyoto-u.ac.jp

Type of the article: Original Research

Author affiliations and contact information:

Takahide Kakigi, MD, Tomohisa Okada, MD, PhD, Mitsunori Kanagaki, MD, PhD, Akira Yamamoto, MD, PhD, Yasutaka Fushimi, MD, PhD, Ryo Sakamoto, MD, and Kaori Togashi, MD, PhD

Department of Diagnostic Imaging and Nuclear Medicine, Kyoto University

Graduate School of Medicine, 54 Shogoin Kawahara-cho, Sakyo-ku, Kyoto, 606-8507, Japan

Phone: +81-75-751-3760, FAX: +81-75-771-9709

E-mail addresses:

tkakigi@kuhp.kyoto-u.ac.jp (Takahide Kakigi)

tomokada@kuhp.kyoto-u.ac.jp (Tomohisa Okada)

mitsuk@kuhp.kyoto-u.ac.jp (MitsunoriKanagaki)

yakira@kuhp.kyoto-u.ac.jp (Akira Yamamoto)

yfushimi@kuhp.kyoto-u.ac.jp (Yasutaka Fushimi) 
ryoskmt@kuhp.kyoto-u.ac.jp (Ryo Sakamoto)

nmdioffice@kuhp.kyoto-ac.jp (Kaori Togashi)

Yoshiki Arakawa, MD, PhD and Jun C. Takahashi, MD, PhD

Department of Neurosurgery, Kyoto University Graduate School of Medicine,

54 Shogoin Kawahara-cho, Sakyo-ku, Kyoto, 606-8507, Japan.

Phone: +81-75-751-3760, FAX: +81-75-771-9709

E-mail addresses:

yarakawa@kuhp.kyoto-u.ac.jp (Yoshiki Arakawa)

juntak@kuhp.kyoto-u.ac.jp (Jun C. Takahashi)

Yoshiki Mikami, MD, PhD

Department of Diagnostic Pathology, Kyoto University Graduate School of

Medicine, 54 Shogoin Kawahara-cho, Sakyo-ku, Kyoto, 606-8507, Japan.

Phone: +81-75-751-3760, FAX: +81-75-771-9709

E-mail address: mika@kuhp.kyoto-u.ac.jp

Taro Shimono, MD, PhD 
Department of Radiology, Osaka City University Graduate School of Medicine, 1-4-3 Asahi-machi, Abeno-ku, Osaka 545-8585, Japan.

Phone: +81-6-6645-3831, Fax: +81-6-6646-6655

e-mail: shimono-knk@umin.ac.jp 


\section{ABSTRACT}

INTRODUCTION: Quantitative values of CT attenuation, apparent diffusion coefficient (ADC) and standardized uptake value (SUV) were investigated for differentiation between pineal parenchymal tumors (PPTs) and germinomas. Differences in age, sex and calcification pattern were also evaluated.

METHODS: Twenty-three patients with PPTs and germinomas in 20 years were retrospectively enrolled under approval of institutional review board. CT attenuation, ADC and SUV (20, 13 and 10 patients, respectively) were statistically compared between the two tumors. Differences in sex and patterns of calcification (“exploded” or “engulfed”) were also examined. Mean patient ages were compared among three groups of pineoblastoma, pineal parenchymal tumor of intermediate differentiation (PPTID) \& pineocytoma and germinoma.

RESULTS: None of the quantitative values of CT attenuation, ADC and SUV showed significant differences between PPTs and germinomas $(p>$.05). However, there was significant difference in age $(p<.05)$ among three groups of pineoblastoma (mean age \pm standard deviation: $7.0 \pm 8.7$ years), PPTID \& pineocytoma (53.7 \pm 11.4 years) and germinoma (19.1 \pm 8.1 years). Sex also showed significant differences between PPTs 
and germinomas $(p=.039)$. Exploded pattern of calcification was found in 9 of the 11 PPT patients, and engulfed pattern in 7 of 9 patients with germinomas. No reverse pattern was observed, and the patterns of calcification were considered highly specific of tumor types.

CONCLUSIONS: None of the quantitative imaging values could differentiate PPTs from germinomas. Age, sex, and calcification patterns were confirmed useful in differentiating these tumors to some degree.

Keywords: Pineal tumor; Germinoma; CT attenuation; Apparent diffusion coefficient; Standardized uptake value. 
Tumors of the pineal region are uncommon and account for approximately $0.4-1 \%$ of all intracranial tumors in American and European literatures, but higher incidence of up to $3.2 \%$ is reported from Japan[1]. Pineal tumors consist of a very heterogeneous group of tumors including germ cell tumors (GCTs) (40\%), pineal parenchymal tumors (PPTs) (14 - 27\%) and neuroepithelial tumors, such as astrocytoma, ependymoma and papillary tumor of the pineal region[2,3,1]. In GCTs, germinoma accounts for the majority of intracranial GCTs (68 - 86\%)[2,4,5], which can be treated successfully with a combination of chemotherapy and radiotherapy. Therefore, biopsy is sufficient for diagnosis of germinoma[6], which is different from PPTs that are categorized into 3 subtypes: pineocytoma (grade 1), pineal parenchymal tumor of intermediate differentiation (PPTID) (grade 2 or 3) and pineoblastoma (grade 4)[3,7]. Surgical resection is mainly selected for pineocytoma occasionally combined with adjuvant chemotherapy and/or radiotherapy[8]. Treatment of PPTID varies from surgery to radiotherapy (radiosurgery or craniospinal irradiation) alone and chemotherapy[9,10]. Standard care of pineoblastoma includes maximal surgical resection with adjuvant craniospinal radiation and systemic chemotherapy[11]. Therefore, differentiation between germinoma and PPTs before surgical procedure is highly important.

For differentiation between PPTs and germinomas, age, sex and patterns of 
calcification, i.e. “exploded” or “engulfed”, have been reported useful[4,2,12], and physiological pineal calcifications are frequently observed on CT[13]. Other imaging characteristics of these tumors at the pineal region have been described on CT and MRI, but their qualitative evaluations could not differentiate the two entities[14,15]. However, there have been only a few studies on ${ }^{18} \mathrm{~F}$-fluoro-2-deoxyglucose-positron emission tomography (FDG-PET). There were only two case reports on FDG-PET findings in pineoblastoma[16] and mixed GCT[17]. No comprehensive imaging study including all conventional imaging methods of CT, MRI and FDG-PET has been conducted to differentiate germinomas and PPTs.

The purpose of this study was to make a comprehensive evaluation on differential capability between germinomas and PPTs using quantitative values of CT attenuation, apparent diffusion coefficient (ADC) and standardized uptake value (SUV). We also evaluated their age, sex and patterns of calcification as "exploded” or "engulfed”, if calcification was present on CT.

\section{MATERIALS AND METHODS}

\section{Patients}

This study was approved by the institutional review board. Informed consent was 
waived due to retrospective nature of this study. The institutional medical recording system and the pathology data base were searched for patients who had CT, MR and FDG-PET imaging with histological verification of germinoma and PPTs between January 1993 and December 2012. Twenty-three patient records (12 females and 11 males; mean 31 years old, ranged from 1 to 68 years) were found and analyzed for this study. Twelve patients had PPTs (5 pineocytomas, 4 PPTIDs and 3 pineoblastomas), and 11 patients had pure germinomas. Imaging was conducted using CT, MRI and FDG-PET in 23, 14 and 10 patients, respectively, but 3 and 1 patients were excluded from CT and MRI, respectively, because they were scanned after biopsy. Patient characteristics are summarized in Table 1.

\section{CT Imaging}

CT images were obtained with a 64-channel multi-detector row CT (MDCT) scanner for 5 patients, a 16-channel MDCT scanner for 4 patients (Aquilion 64 and 16, respectively, Toshiba Medical Systems, Otawara, Japan), and a 4-channel MDCT scanner for 11 patients (W-3000, Hitachi Medical Co, Tokyo, Japan). Resolutions of all CT images were $0.4 \mathrm{~mm} \times 0.4 \mathrm{~mm} \times 7-8 \mathrm{~mm}$. 


\section{MR Imaging}

MR scans were conducted with a 3T scanner for 6 patients and a $1.5 \mathrm{~T}$ scanner for 5 patients (MAGNETOM Trio and MAGNETOM Symphony, respectively, Siemens Medical Systems, Erlangen, Germany). The other 1.5T scanner was used for 2 patients (Signa Genesis, GE Medical Systems, Milwaukee, Wisconsin, USA). In addition to axial T1-weighted, T2-weighted and FLAIR images, contrast-enhanced axial T1-weighted images were acquired after administration of a Gadolinium contrast agent (0.1 mmol/kg). Diffusion weighted imaging (DWI) was acquired with the following parameters: TR $=3700$ - $10000 \mathrm{~ms}$; TE $=80$ - $101 \mathrm{~ms}$; slice thickness $=3$ - 5 mm; slice spacing = $1-1.8 \mathrm{~mm}$; pixel spacing $=0.9-1.7 \mathrm{~mm} \times 0.9-1.7 \mathrm{~mm} ; \mathrm{FOV}=220 \mathrm{~mm} \times$ $220 \mathrm{~mm}$. DWI was conducted with 2 different motion-probing gradient values $(b=0$ and $1000 \mathrm{~s} / \mathrm{mm}^{2}$ ) applied in 3 orthogonal directions. The acquired images were combined to form a single composite DWI and their apparent diffusion coefficient (ADC) values were calculated for each pixel to make ADC images.

\section{FDG-PET Imaging}

FDG-PET scans were conducted using a PET/CT scanner for 7 patients and a PET scanner for 3 patients (Discovery ST Elite and Advance, respectively, GE Healthcare, 
Waukesha, WI, USA). Patients fasted for at least 4 hours prior to the scans. After intravenous administration of $4 \mathrm{MBq} / \mathrm{kg}$ of FDG, patients rested in a waiting room for 30 minutes. Emission scans of the brain were conducted for 15 minutes with $128 \times 128$ matrix and 47 slices and $128 \times 128$ matrix and 35 slices, resulting in resolutions of 2.0 $\mathrm{mm} \times 2.0 \mathrm{~mm} \times 3.27 \mathrm{~mm}$ and $2.0 \mathrm{~mm} \times 2.0 \mathrm{~mm} \times 4.25 \mathrm{~mm}$, respectively for the scanners.

\section{Data Analysis}

All images were evaluated independently by 2 experienced neuroradiologists (T.K. and R.S., both had experience in diagnostic imaging for 9 years) blinded to clinical data. Region of interests (ROIs) for the tumors were defined on an analysis console (Advantage Workstation 4.4, GE Medical Systems, Milwaukee, Wisconsin, USA) to measure maximum and mean non-enhanced CT attenuation values (CTmax and CTmean, respectively), mean and minimum ADC values (ADCmean and ADCmin, respectively) and mean and maximum SUV (SUVmean and SUVmax, respectively). ROIs were drawn on one slice with the largest enhancement area by referring to the MR or CT images. Regions suggestive of a cystic change, necrosis or calcification were excluded from ROIs. When only FDG-PET imaging existed (1 patient of pineoblastoma 
and 1 patient of germinoma), ROIs were drawn by referring to FDG uptakes. We evaluated presence and pattern of calcification as "exploded” or “engulfed” on axial non-enhanced CT.

\section{Statistical Analysis}

Inter-observer variability was evaluated using intraclass correlation coefficient (ICC)[18] for measuring the quantitative values and ROI sizes. The measured values of two evaluators were averaged and compared between germinoma and PPTs using Mann-Whitney test. Age difference was compared among 3 groups of germinomas, pineoblastomas and PPTIDs \& pineocytomas using Kruskal-Wallis test, because the pineoblastoma is found in much younger patients than the pineocytoma or PPTID. Sex difference was examined with Fisher's exact test. A $p$ value less than .05 was considered statistically significant. Statistical analysis was conducted with a commercially available statistical software package (MedCalc Version 12.5.0, MedCalc Software, Mariakerke, Belgium).

\section{RESULTS}

ICCs were 0.76 to 0.99 , which was recognized as good to excellent inter-observer 
reproducibility[19] for all the quantitative values and ROI sizes. Statistically significant difference was not found between germinoma and PPTs for any of the measured quantitative values $(p=.17, .08, .72, .09, .61$ and .91 respectively for CTmax, CTmean, ADCmean, ADCmin, SUVmean and SUVmax; see Tables 1 \& 2 and Figs. 1 - 4).

Calcification was detected in 9 of 12 patients (75\%) with PPTs, and all cases showed "exploded” calcification. Seven of 11 patients with germinoma had calcification, and "engulfed" calcification was found in all cases (Fig. 4). No discrepancy was observed between the observers.

The mean ages \pm standard deviations were $19.1 \pm 8.1,7.0 \pm 8.7$ and $53.7 \pm 11.4$ years old for patients with germinoma, pineoblastoma and PPTIDs \& pineocytomas, respectively. When these 3 groups were compared, significant difference was observed among the all 3 groups ( $p<.05$, post-hoc analysis, Fig. 5). Eight out of 11 patients were male in germinoma, and 9 out of 12 were female in PPTs. The difference was statistically significant $(p=.039)$.

\section{DISCUSSION}

This is the first study that investigated quantitative imaging values of CT attenuation, ADC and SUV, comprehensively, for differentiation between germinomas and PPTs. 
The imaging spectrum of these tumors has been described[4,12,14,15,2], but most findings are limited to CT or MRI. There are two case reports on its very high uptake by FDG-PET imaging in pineoblastoma[16] and little uptake in mixed GCT[17], but no report exists on pineocytoma and PPTID on FDG-PET. Moreover, no comparison has been conducted comprehensive of all modalities between PPT and germinoma, which was conducted in this study. We focused on pure germinoma, because GCTs except germinoma can be generally differentiated using serum and cerebrospinal fluid (CSF) levels of tumor-produced oncoproteins ( $\alpha$-fetoprotein, $\beta$-human chorionic gonadotropin ( $\beta$-hCG), placental alkaline phosphatase)[2].

On CT, the pineoblastoma and germinoma are frequently recognized as hyper-attenuating masses, which reflect highly cellular histologic features compared with the pineocytoma and PPTID[2]. However, the difference in CT value between the germinoma and PPT was around 3 Hounsfield unit (HU) (see Table 2), which was not statistically significant.

Dumrongpisutikul et al.[20] have proposed the utility of ADC values between the germinoma and PPT. They found that germinoma had higher ADC values than the pineal cell tumors, however, we found no significant difference in ADC values between PPTs and germinoma. They drew ROIs covering the entire lesions inclusive of cystic or 
necrotic regions, while we put them on areas with contrast enhancement because such region size may vary. The ADC value of the solid portion of the tumor is considered to have limited role in making differential diagnosis.

In FDG-PET, no study that compared between PPTs and germinomas has been reported. This is the first study to investigate the difference, but no significant difference was found between germinomas and PPTs. In average, Germinomas had higher SUVs than PPTs, though two cases of pineocytoma had very high SUVs (see Table 1). The pineocytoma is a low-grade tumor and may retain functionality. FDG uptake reflects not only cell density and mitotic activity of pineal tumors, but also other activities such as regulation of certain circulating hormone levels and short-term (e.g., diurnal or circadian) biologic rhythm[13]. Such complex activity of the pineal tumor may result in failure of differentiation using SUV values.

It has already been reported that germinomas “engulf” physiological calcifications, whereas PPTs scatter or "explode” calcifications[4,2]. When calcification existed, the pattern classification was considered to have high differentiation capability of the two. In this study, these two tumors were completely differentiated by the patterns with no disagreement between the two evaluators. However, calcification was detected in $75 \%$ on non-enhanced CT. Other clues for differentiation might be required. 
The pineocytoma and PPTID occur predominantly in adults from the third to sixth decades of age[13], whereas the pineoblastoma most commonly occurs in the first 2 decades[7]. Ninety percent of patients with the germinoma are less than 20 years old[4,7]. The mean ages of the 3 tumor groups were statistically significant, but there was large overlap between pineoblastoma and germinoma (see Fig. 5), and differentiation was considered difficult between them.

No gender predilection is reported in pineocytoma and pineoblastoma, but a slight female preponderance is reported in PPTID[7,2]. Germinoma in the pineal region are 10 times more common in males[7]. In this study, all of PPTID patients were female, and male predominance was found in germinoma. Statistically, there was significant difference between PPT and germinoma in sex. However, there were 3 female patients out of 11 germinoma patients, and its differentiation from pineoblastoma is considered difficult.

There is a limitation in this retrospective study that the numbers of patients with PPTs and germinomas were relatively small, which is inevitable considering relative rarity of these tumors. As the other limitation, we have to consider errors caused by difference in MR imaging methods, such as magnetic field strengths (i.e. 1.5T vs. 3T), MR vendors and imagers for measuring the ADC values, which were 4 - 9\%, 7\% and up 
to $8 \%$, respectively [21]. ADCmin had weak tendency $(p=0.09)$ to differentiate PPT from germinoma. Variability in MR imaging methods might obscure the potentially existing difference between these two tumors, but images only form a single MR imaging method may not be technically feasible due to the aforementioned reason.

\section{CONCLUSIONS}

Roles of quantitative values of CT attenuation, ADC and SUV were investigated in differentiating between PPTs and germinomas, but none was found useful. However, as was previously reported, age, sex and calcification patterns had statistically significant differences and were confirmed useful in differentiating these tumors to some degree. In PPTs, we may distinguish pineocytomas and PPTIDs from pineoblastomas, because their age distributions are highly different. However, differentiation between pineoblastomas and germinomas is difficult, when there is no calcification.

\section{Conflict of Interest}

All the authors confirm that there is no conflict of interest.

\section{Disclosure}


All the authors have nothing to disclose related to this article.

\section{REFERENCES}

1. Senft C, Raabe A, Hattingen E, Sommerlad D, Seifert V, Franz K (2008) Pineal

parenchymal tumor of intermediate differentiation: diagnostic pitfalls and discussion of treatment options of a rare tumor entity. Neurosurg Rev 31 (2):231-236.

doi:10.1007/s10143-008-0126-8

2. Smith AB, Rushing EJ, Smirniotopoulos JG (2010) From the archives of the AFIP: lesions of the pineal region: radiologic-pathologic correlation. Radiographics 30 (7):2001-2020. doi:10.1148/rg.307105131

3. Han SJ, Clark AJ, Ivan ME, Parsa AT, Perry A (2011) Pathology of pineal parenchymal tumors. Neurosurg Clin N Am 22 (3):335-340, vii. doi:10.1016/j.nec.2011.05.006

4. Gaillard F, Jones J (2010) Masses of the pineal region: clinical presentation and radiographic features. Postgrad Med J 86 (1020):597-607. doi:10.1136/pgmj.2009.087460 5. Horowitz MB, Hall WA (1991) Central nervous system germinomas. A review. Arch Neurol $48(6): 652-657$

6. Lekovic GP, Gonzalez LF, Shetter AG, Porter RW, Smith KA, Brachman D, Spetzler RF 
(2007) Role of Gamma Knife surgery in the management of pineal region tumors. Neurosurg

Focus 23 (6):E12. doi:10.3171/FOC-07/12/E12

7. Louis D, Ohgaki H, Wiestler O, Cavenee W (2007) Who Classification of Tumours of the Central Nervous System 4th edition. World Health Organization

8. Wilson DA, Awad AW, Brachman D, Coons SW, McBride H, Youssef E, Nakaji P, Shetter AG, Smith KA, Spetzler RF, Sanai N (2012) Long-term radiosurgical control of subtotally resected adult pineocytomas. J Neurosurg 117 (2):212-217. doi:10.3171/2012.5.JNS1251

9. Pusztaszeri M, Pica A, Janzer R (2006) Pineal parenchymal tumors of intermediate differentiation in adults: case report and literature review. Neuropathology 26 (2):153-157 10. Fauchon F, Jouvet A, Paquis P, Saint-Pierre G, Mottolese C, Ben Hassel M, Chauveinc L, Sichez JP, Philippon J, Schlienger M, Bouffet E (2000) Parenchymal pineal tumors: a clinicopathological study of 76 cases. Int J Radiat Oncol Biol Phys 46 (4):959-968 11. Tate MC, Rutkowski MJ, Parsa AT (2011) Contemporary management of pineoblastoma. Neurosurg Clin N Am 22 (3):409-412, ix. doi:10.1016/j.nec.2011.05.001 12. Ganti SR, Hilal SK, Stein BM, Silver AJ, Mawad M, Sane P (1986) CT of pineal region tumors. AJR Am J Roentgenol 146 (3):451-458. doi:10.2214/ajr.146.3.451 13. Smirniotopoulos JG, Rushing EJ, Mena H (1992) Pineal region masses: differential diagnosis. Radiographics 12 (3):577-596 
14. Korogi Y, Takahashi M, Ushio Y (2001) MRI of pineal region tumors. J Neurooncol 54

(3):251-261

15. Reis F, Faria AV, Zanardi VA, Menezes JR, Cendes F, Queiroz LS (2006) Neuroimaging in pineal tumors. J Neuroimaging 16 (1):52-58. doi:10.1177/1051228405001514

16. Bakheet SM, Hassounah M, Al-Watban J, Homsi M, Powe J, Larsson S (1999) F-18 FDG PET scan of a metastatic pineoblastoma. Clin Nucl Med 24 (3):198-199

17. Park SA, Kim TY, Choi SS, Yang CY, Kim HS, Choi KH (2012) (1)(8)F-FDG PET/CT

imaging for mixed germ cell tumor in the pineal region. Clin Nucl Med 37 (3):e61-63.

doi:10.1097/RLU.0b013e31823926fc

18. Carrasco JL, Jover L (2003) Estimating the generalized concordance correlation

coefficient through variance components. Biometrics 59 (4):849-858

19. Busing KA, Kilian AK, Schaible T, Debus A, Weiss C, Neff KW (2008) Reliability and validity of MR image lung volume measurement in fetuses with congenital diaphragmatic hernia and in vitro lung models. Radiology 246 (2):553-561. doi:10.1148/radiol.2462062166

20. Dumrongpisutikul N, Intrapiromkul J, Yousem DM (2012) Distinguishing between germinomas and pineal cell tumors on MR imaging. AJNR Am J Neuroradiol 33 (3):550-555. doi:10.3174/ajnr.A2806

21. Sasaki M, Yamada K, Watanabe Y, Matsui M, Ida M, Fujiwara S, Shibata E; Acute 
Stroke Imaging Standardization Group-Japan (ASIST-Japan) Investigators (2008)

Variability in absolute apparent diffusion coefficient values across different platforms may

be substantial: a multivendor, multi-institutional comparison study. Radiology 249

(2):624-30. doi: 10.1148/radiol.2492071681. 
TABLES.

Table 1. Patient characteristics and quantitative imaging values

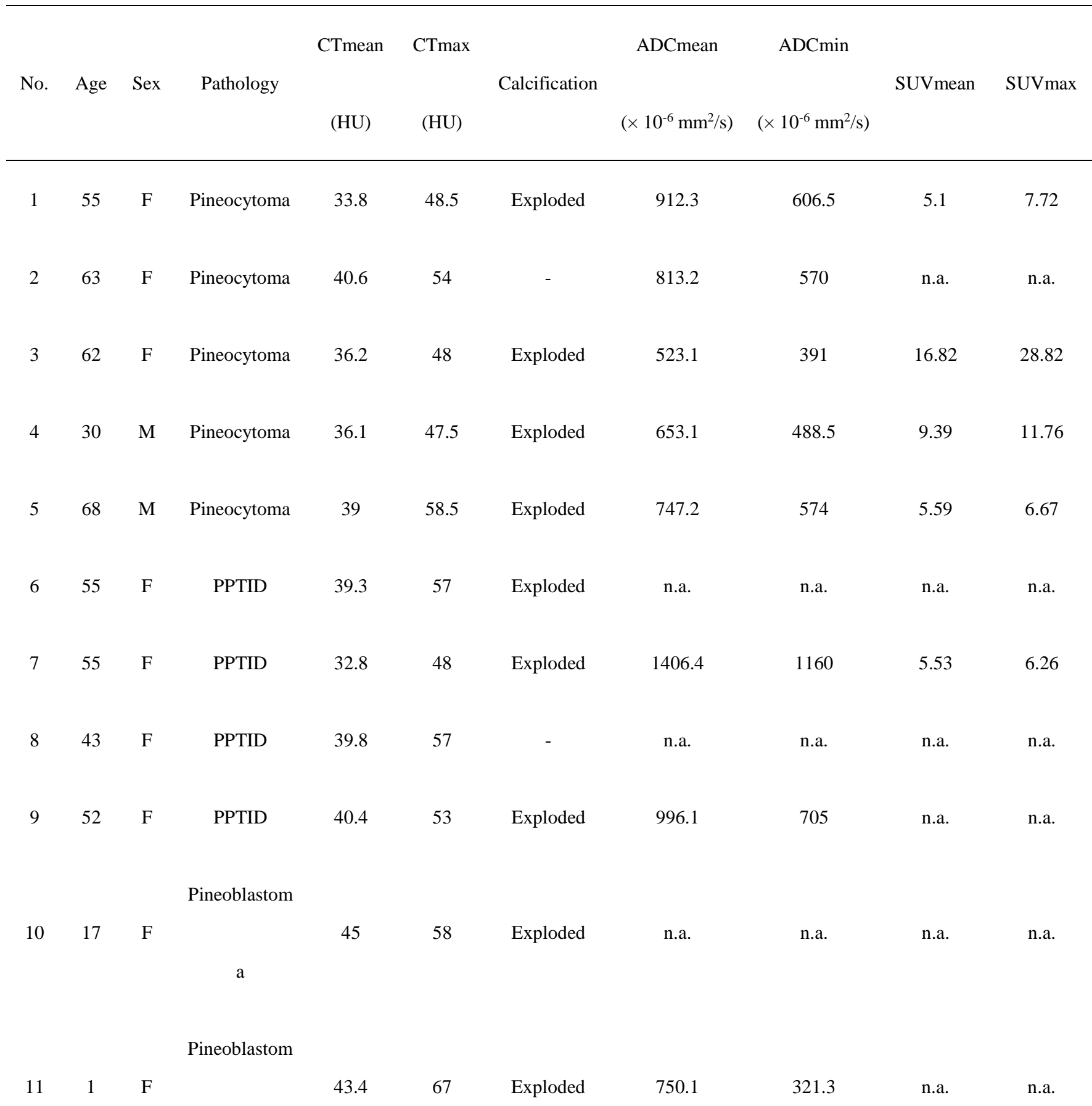




\section{Pineoblastom}

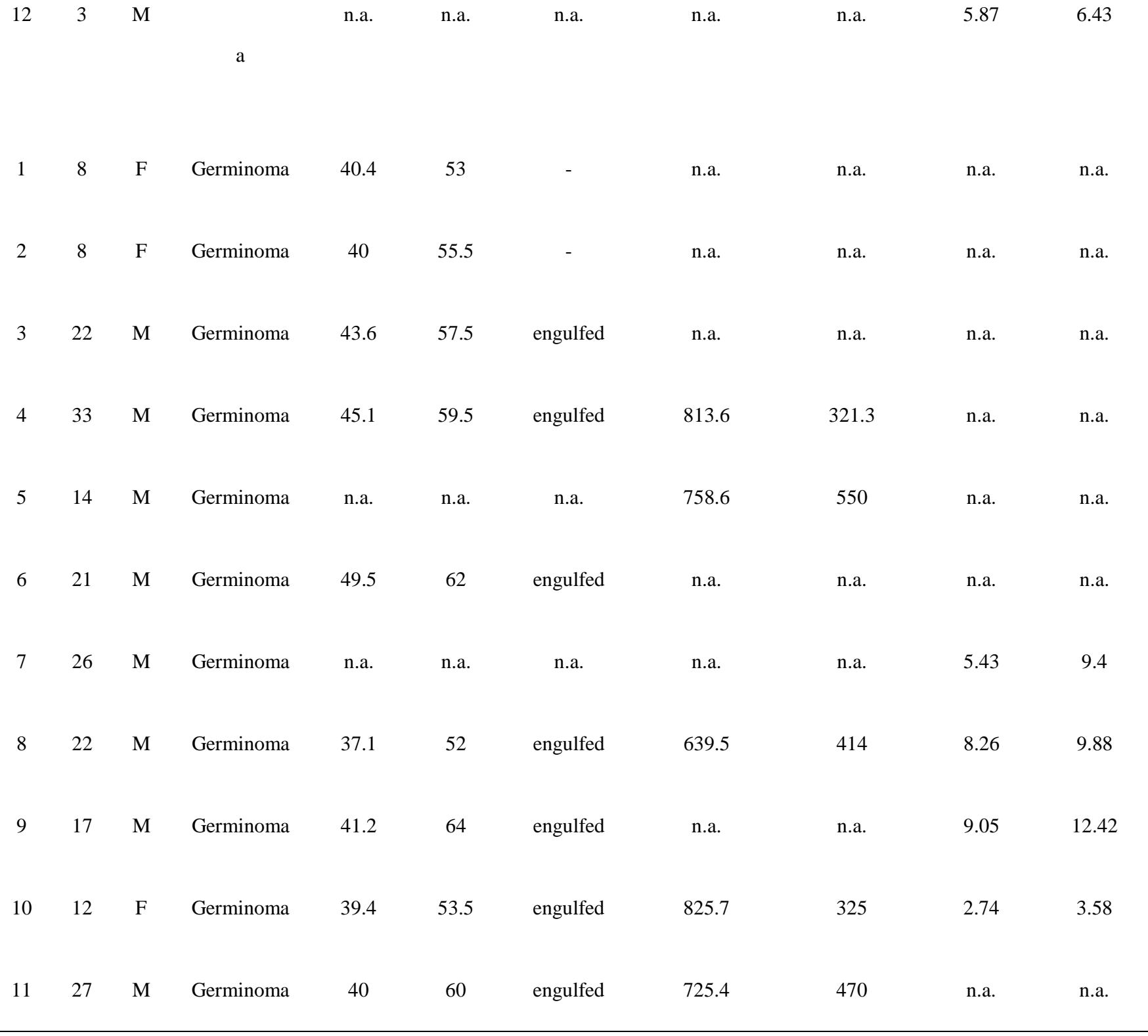

Note: $\mathrm{ADC}=$ apparent diffusion coefficient, $\mathrm{HU}=$ Hounsfield unit, n.a. $=$ not available, PPTID $=$ pineal parenchymal tumor of

intermediate differentiation, SUV = standardized uptake value. 
Table 2. Differences between PPT and germinoma in CTmax, CTmean, ADCmean, ADCmin, SUVmean and SUVmax

\begin{tabular}{|c|c|c|c|c|}
\hline \multirow{3}{*}{ Quantitative } & & PPT & $\mathrm{G}$ & \multirow{4}{*}{$p$ value } \\
\hline & \multirow{3}{*}{$\mathrm{n}$} & median & median & \\
\hline & & & & \\
\hline values & & (interquartile range) & (interquartile range) & \\
\hline CTmax (HU) & 20 & $54.0(48.1-57.8)$ & $57.5(53.4-60.5)$ & .17 \\
\hline CTmean (HU) & 20 & $39.3(36.1-40.6)$ & $40.4(39.8-44.0)$ & .08 \\
\hline \multicolumn{5}{|l|}{ ADCmean } \\
\hline & 13 & $781.6(700.1-954.2)$ & 758.6 (703.9-816.6) & .72 \\
\hline \multicolumn{5}{|l|}{$\left(\times 10^{-6} \mathrm{~mm}^{2} / \mathrm{s}\right)$} \\
\hline \multicolumn{5}{|l|}{ ADCmin } \\
\hline & 13 & 572.0 (439.8-655.8) & $414.0(324.1-490.0)$ & .09 \\
\hline \multicolumn{5}{|l|}{$\left(\times 10^{-6} \mathrm{~mm}^{2} / \mathrm{s}\right)$} \\
\hline SUVmean & 10 & 5.73 (5.53-9.39) & $6.85(4.09-8.66)$ & .61 \\
\hline SUVmax & 10 & $7.20(6.43-11.76)$ & 9.64 (6.49-11.15) & .91 \\
\hline
\end{tabular}

Note: ADC = apparent diffusion coefficient, $\mathrm{G}=$ germinoma, $\mathrm{HU}=$ Hounsfield unit, $\mathrm{PPT}=$ pineal parenchymal tumor, $\mathrm{SUV}=$ 


\section{FIGURE}

Fig 1. Plots of quantitative values for comparisons between PPT and germinoma. No significant difference was observed between them. The long horizontal bars indicate median values, and the short horizontal bars show interquartile range.

Note: $\mathrm{ADC}=$ apparent diffusion coefficient, $\mathrm{HU}=$ Hounsfield unit, $\mathrm{PPT}=$ pineal parenchymal tumor, SUV = standardized uptake value
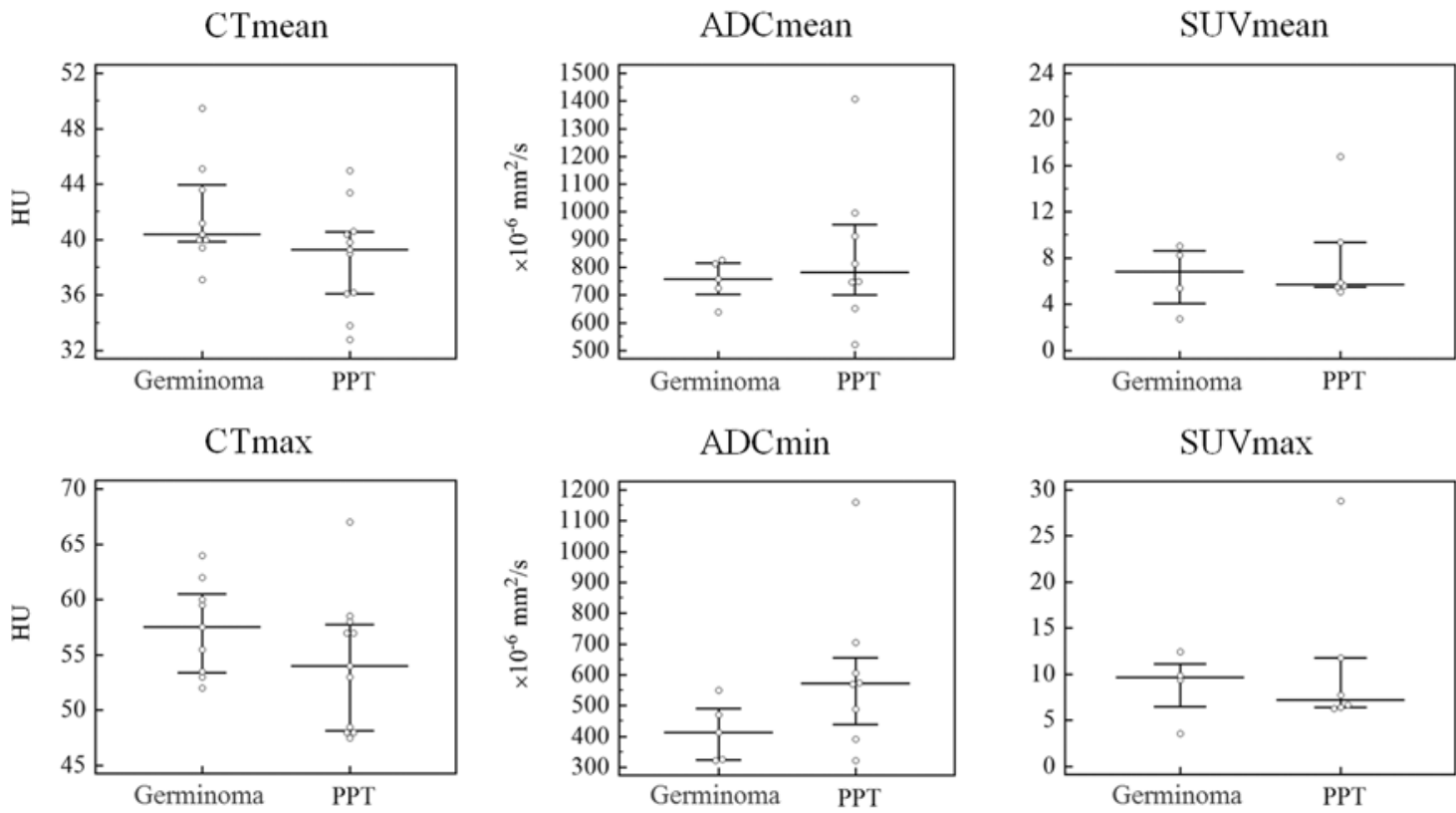

SUVmax

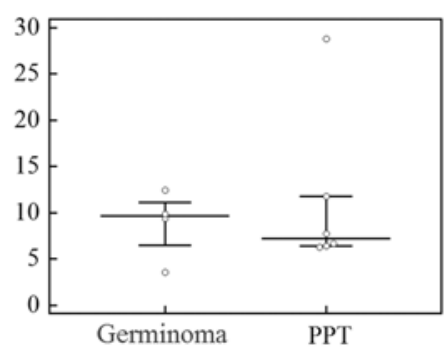


Fig 2. Diffusion-weighted images of pathologically proven (A) pineocytoma, (B) PPTID, (C) pineoblastoma and (D) germinoma. They have minimum ADC values of 574, 705, 343 and $325 \times 10^{-6} \mathrm{~mm}^{2} / \mathrm{s}$, respectively.

Note: $\mathrm{ADC}=$ apparent diffusion coefficient, PPTID = pineal parenchymal tumor of intermediate differentiation
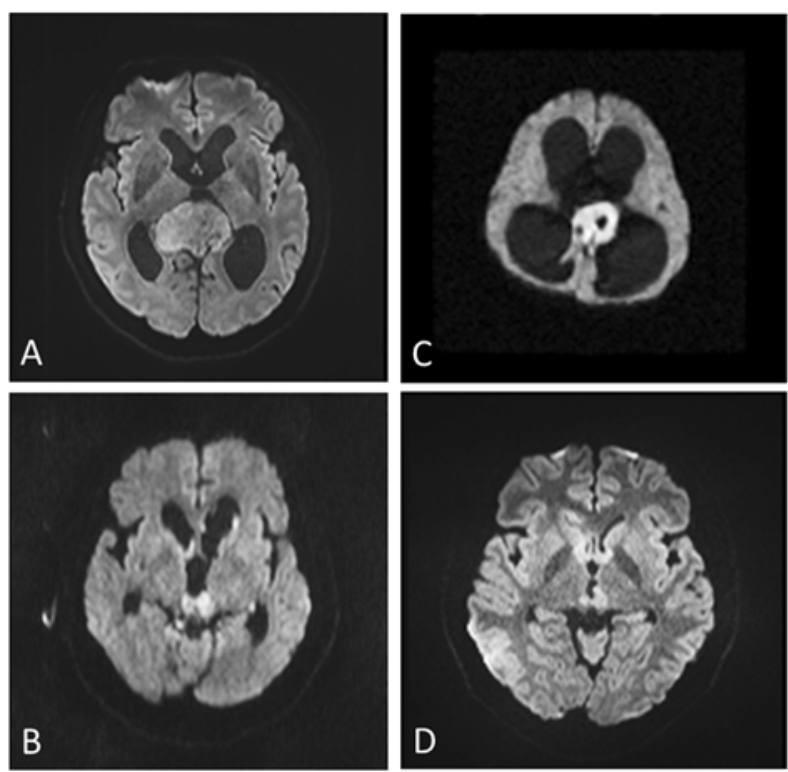
Fig 3. FDG-PET images of (A) pineocytoma, (B) PPTID, (C) pineoblastoma and (D) germinoma. They have maximum SUV values of 6.67, 6.26, 6.43 and 9.88, respectively.

Note: PPTID = pineal parenchymal tumor of intermediate differentiation, SUV = standardized uptake value
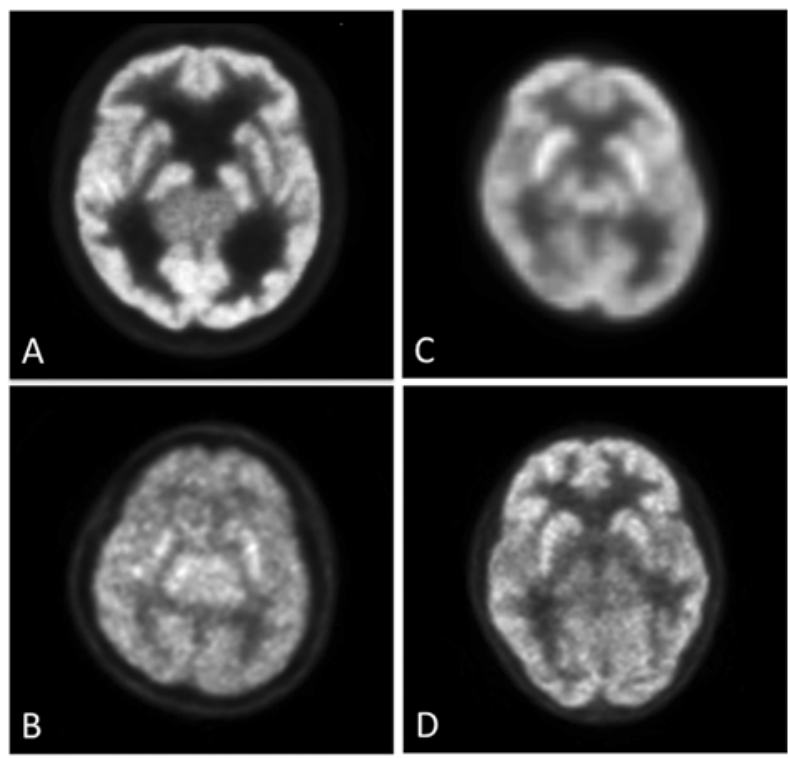
Fig 4. CT images of pathologically proven (A) pineocytoma, (B) PPTID, (C)

pineoblastoma and (D) germinoma. They have mean CT values of 36.1, 32.8, 45.0 and

49.5 HU, respectively. Cases A, B and C show "exploded” calcfication, whereas case D shows “engulfed” calcification.

Note: HU = Hounsfield unit, PPTID = pineal parenchymal tumor of intermediate differentiation

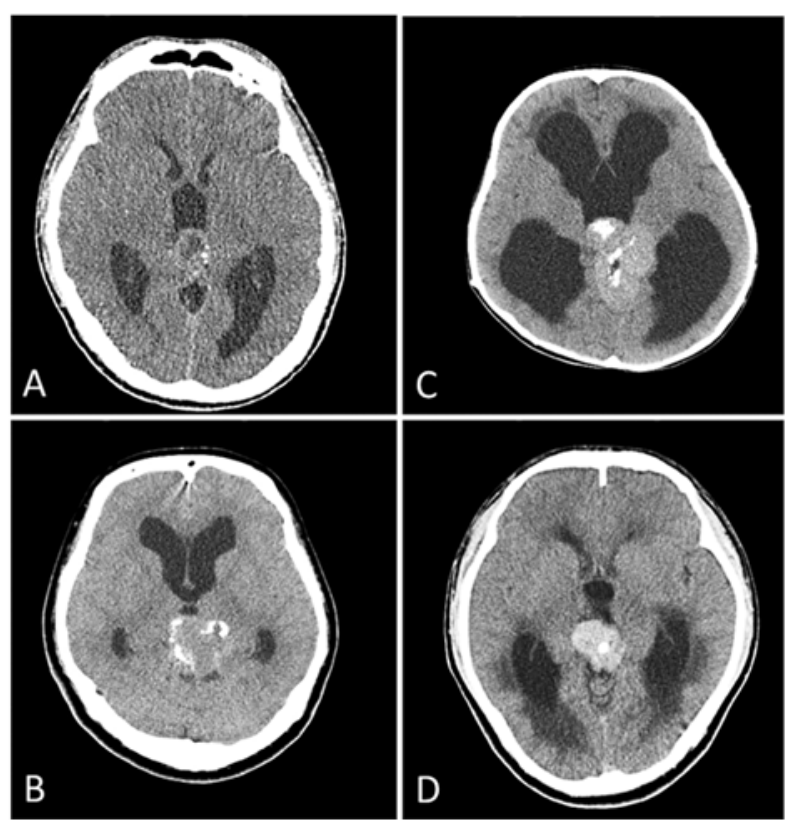


Fig 5. Age difference among germinomas, pineoblastomas and PPTIDs \& pineocytomas. Significant difference is observed in all pairs of the three groups $(p<.05$, post-hoc analysis). The long horizontal bars indicate mean values, and the short horizontal bars show 95\% confidence intervals.

Note: $\mathrm{PB}=$ pineoblastoma, $\mathrm{PC}=$ pineocytoma, $\mathrm{PPTID}=$ pineal parenchymal tumor of intermediate differentiation

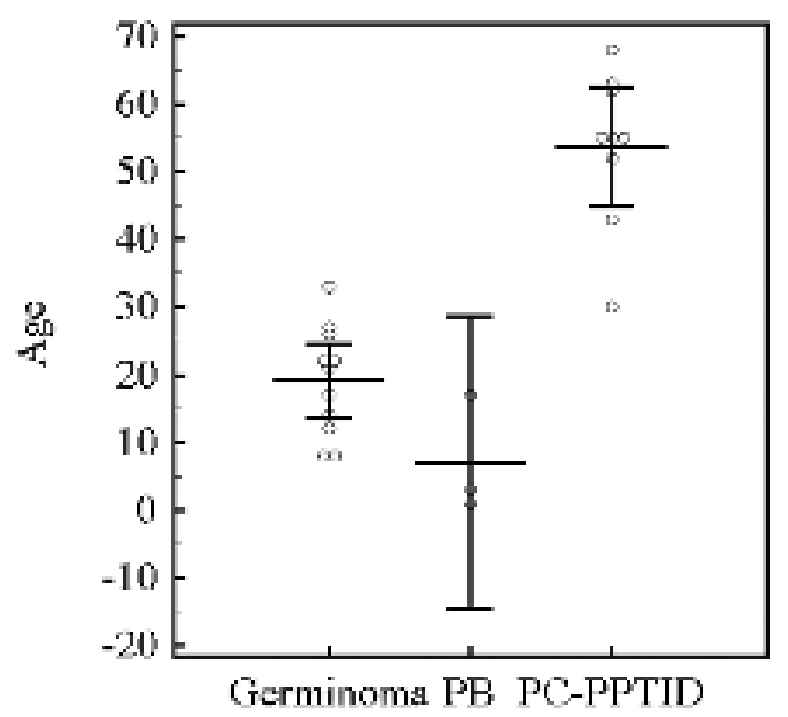

